\title{
Manifestações otoneurológicas associadas à terapia anti-retroviral
}

\author{
Otoneurological manifestations associated \\ with antiretroviral therapy
}

\author{
Andrêza Batista Cheloni Vieira1, Dirceu Bartolomeu Greco ${ }^{1,2}$, \\ Márcia Miliane Maciel Teófilo ${ }^{3}$ e Denise Utsch Gonçalves ${ }^{1,3}$
}

\begin{abstract}
RESUMO
Ototoxicidade e terapia anti-retroviral parecem estar associadas. 0 objetivo desse estudo foi avaliar essa possível correlação. Foram avaliados 779 prontuários médicos de pacientes infectados pelo HIV e regularmente acompanhados, sendo 162 tratados com terapia anti-retroviral e 122 não tratados (controle). Pacientes em tratamento eram mais velhos (média 42 anos), com maior tempo de confirmação sorológica (80 meses) e com menor carga viral $(\mathrm{p}=0,00)$. CD $4+$ foi semelhante entre os grupos $(\mathrm{P}=0,60)$. No grupo tratado, três $(1,8 \%)$ casos de perda auditiva idiopática $\mathrm{e}$ dois $(1,3 \%)$ de perda auditiva relacionada a otosclerose foram observadas e ambas iniciadas após terapia anti-retroviral. Nenhuma diferença estatística relacionada à perda auditiva idiopática foi encontrada entre os grupos. Enquanto estudos descritivos consideram possível ototoxidade associada à terapia anti-retroviral, esse possível efeito adverso não foi relacionado à terapia anti-retroviral neste estudo. Contrariamente, otosclerose poderia estar correlacionada à terapia anti-retroviral. Este assunto merece ser estudado.
\end{abstract}

Palavras-chaves: Perda auditiva. Tontura. Zumbido. Infecções por HIV. Terapia anti-retroviral de alta atividade.

\begin{abstract}
Ototoxicity and antiretroviral therapy seem to be associated. The aim of this study was to evaluate this possible correlation. Evaluations were carried out on 779 medical records from HIV-infected patients who were being regularly followed up, of whom 162 were being treated with antiretroviral therapy and 122 were untreated (controls). The patients undergoing treatment were older (mean: 42 years), had had serological confirmation for longer times ( 80 months) and had smaller viral loads $(P=0.00)$. CD4+ was similar between the groups $(P=0.60)$. In the treated group, three cases (1.8\%) of idiopathic hearing loss and two (1.3\%) of otosclerosis-related hearing loss were observed, which both started after antiretroviral therapy. No statistical difference relating to idiopathic hearing loss was found between the groups. While descriptive studies consider possible ototoxicity associated with antiretroviral therapy, this possible adverse effect was not related to the antiretroviral therapy in this study. Conversely, otosclerosis might have been correlated with antiretroviral therapy. This issue deserves to be studied.
\end{abstract}

Key-words: Hearing loss. Dizziness. Tinnitus. HIV infections. Highly active antiretroviral therapy.

A associação entre a infecção pelo vírus da imunodeficiência humana (HIV) e sinais e sintomas otoneurológicos (perda de audição, zumbido e tontura) tem se tornado objeto de estudos nacionais e internacionais ${ }^{2571230}$

As causas das alterações otoneurológicas vêm sendo associadas às infecções oportunistas, às drogas ototóxicas e à ação direta vírus no sistema cocleovestibular ${ }^{125671230}$.
Com o tratamento da infecção pelo HIV, dúvidas surgiram sobre a ação tóxica dos medicamentos anti-retrovirais na orelha interna. A ototoxicidade dessas drogas vem sendo apresentada por vários autores em estudos descritivos com frequiência variando de $1 \%$ a $29 \%^{2411} 172931$. Avaliar de forma comparativa a possível correlação de alterações otoneurológicas e tratamento anti-retroviral torna-se relevante por permitir melhor adequação do esquema terapêutico ao se conhecer os possíveis efeitos colaterais associados.

\footnotetext{
1. Programa de Pós-graduação em Ciências da Saúde: Infectologia e Medicina Tropical, Faculdade de Medicina, Universidade Federal de Minas Gerais, Belo Horizonte, MG 2. Departamento de Clínica Médica, Faculdade de Medicina, Universidade Federal de Minas Gerais, Belo Horizonte, MG. 3. Departamento de Oftalmologia, Otorrinolaringologia e Fonoaudiologia, Faculdade de Medicina, Universidade Federal de Minas Gerais, Belo Horizonte, MG

Endereço para correspondência: Prof ${ }^{a}$ Denise Utsch Gonçalves. Dept ${ }^{\circ}$ de Oftalmologia, Otorrinolaringologia e Fonoaudiologia/FM/UFMG. Av. Alfredo Balena 190/sala 3005, Santa Efigênia, 30130-100 Belo Horizonte, MG

Telefax: $55313248-9767$

e-mail: deniseg@medicina.ufmg.br

Recebido para publicação em: 10/07/2007

Aceito em: 15/01/2008
} 


\section{MATERIAL E MÉTODOS}

Foram selecionados 779 prontuários de pacientes infectados pelo HIV atendidos no Centro de Treinamento e Referência em Doenças Infecciosas e Parasitárias Orestes Diniz (CRT-DIP), em Belo Horizonte. Prontuários selecionados incluíram pacientes: 1)infectados pelo HIV conforme registro do diagnóstico médico; 2) assistidos pelo CRT-DIP; 3) maiores de 18 anos de ambos os sexos; 4) não-tratados ou em tratamento regular com antiretrovirais. Foram excluídos os pacientes cujos prontuários estavam com contagem de CD4+ ausente.

Os prontuários finalmente selecionados foram 284, sendo $162(57 \%)$ de pacientes que usavam anti-retrovirais (grupo de estudo) e 122 (43\%) de pacientes que não estavam em uso dessa medicação (grupo controle). Dessa forma, o desenho do estudo foi do tipo caso-controle, no qual o grupo de caso, composto por pacientes que faziam uso de medicação anti-retroviral foi comparado ao grupo controle, constituído por pacientes sem anti-retrovirais, para avaliar se as manifestações otoneurológicas associam-se a essas drogas.

0 contato com os participantes foi feito através de cartaconvite. Todos os pacientes que responderam à convocação para o estudo com queixa otoneurológica foram submetidos à entrevista em que se procurou averiguar as queixas otológicas, vestibulares e os efeitos colaterais aos medicamentos da terapia anti-retroviral ${ }^{18}{ }^{28}$. A história pregressa relacionada a doenças otológicas e vestibulares foi avaliada. A partir da queixa, os pacientes foram submetidos às avaliações audiológica, otorrinolaringológica e exames complementares para diagnóstico e tratamento adequados.

A hipótese nula considerada nesse estudo foi a de que não existe associação entre o uso de drogas anti-retrovirais e sinais e sintomas otoneurológicos, considerando-se nível de significância de $5 \%$ e poder do estudo de $80 \%$.

Para o cálculo do tamanho da amostra, considerou-se a prevalência de alterações otoneurológicas em pacientes tratados com anti-retrovirais de $20 \%{ }^{23}$ e prevalência em pacientes não tratados com essa medicação de $6,6 \%{ }^{20}$, sendo o tamanho calculado de 114 pacientes para cada grupo 245710121720232931 .

0 presente estudo e seu termo de consentimento livre e esclarecido foram aprovados pelo Comitê de Ética em Pesquisa da Universidade Federal de Minas Gerais (COEP-UFMG) parecer 065/05 e pelo Comitê de Ética em Pesquisa - Secretaria Municipal de Saúde da Prefeitura de Belo Horizonte (CEP-SMSA/PBH) parecer 015/2005.

\section{RESULTADOS}

Dados epidemiológicos e tempo de confirmação sorológica. 0 grupo não-tratado com anti-retrovirais foi composto por $66(54,1 \%)$ homens e $56(45,9 \%)$ mulheres e o grupo tratado composto por $95(58,6 \%)$ homens e $67(41,4 \%)$ mulheres ( $\mathrm{P}=0,41 ;$ OR:1,20; IC: 0,73-1,99).
A média de idade do grupo não-tratado foi de 34,7 anos $(\mathrm{DP}=34,7)$ e mediana de 34 anos, enquanto no grupo tratado, a média de idade foi de 41,8 anos $(\mathrm{DP}=9,3)$ e mediana de $41 \operatorname{anos}(\mathrm{P}=0,00)$.

O tempo médio da data de confirmação sorológica da infecção pelo HIV para o grupo tratado foi de 80,3 meses (desvio padrão de 33,9 meses) e de 39,3 meses (desvio padrão de 31,2 meses) para o grupo não tratado $(\mathrm{P}=0,00)$.

Contagem de linfócitos CD4+ e carga viral. Dos 162 pacientes tratados com anti-retrovirais, 91 (56,2\%) apresentaram contagem de CD4+ entre 200-400 células $/ \mathrm{mm}^{3} \mathrm{e}$ $71(43,8 \%)$ apresentaram essa contagem acima de 500 células/ $\mathrm{mm}^{3}$. Quanto aos 122 pacientes não tratados, 73 (59,8\%) apresentaram contagem de CD4+ entre 200-400 células/ $\mathrm{mm}^{3}$ e $49(40,2 \%)$ apresentaram essa contagem acima de 500 células $/ \mathrm{mm}^{3}(\mathrm{P}=0,60 ; \mathrm{OR}=0,86 \mathrm{IC}=0,52-1,43)$. Nenhum paciente incluído no estudo apresentou contagem abaixo de 200 células $/ \mathrm{mm}^{3}$.

$\mathrm{Na}$ Tabela 1, a carga viral dos pacientes de ambos os grupos estudados é apresentada. 0 registro da carga viral de 12 pacientes do grupo não tratado e de cinco pacientes do grupo tratado não foi encontrado nos prontuários médicos.

Tabela 1 - Distribuição comparativa da carga viral de 267 pacientes infectados pelo HIV com e sem tratamento anti-retroviral. Belo Horizonte, Centro de Treinamento e Referência em Doenças Infecciosas e Parasitárias Orestes Diniz, 2005-2006.

\begin{tabular}{|c|c|c|c|c|c|c|c|}
\hline \multirow{3}{*}{$\begin{array}{l}\text { Carga viral } \\
\text { (cópias de RNA } \\
\text { viral/ml de plasma) }\end{array}$} & \multicolumn{4}{|c|}{ Pacientes infectados pelo HIV } & \multirow{3}{*}{ s } & \multirow{3}{*}{ OR } & \multirow{3}{*}{ IC (95\%) } \\
\hline & \multicolumn{2}{|c|}{$\begin{array}{c}\text { tratados com } \\
\text { anti-retrovirais } \\
\left(n^{0}=157\right)\end{array}$} & \multicolumn{2}{|c|}{$\begin{array}{c}\text { não tratados } \\
\text { com anti-retrovirais } \\
\left(n^{0}=110\right)\end{array}$} & & & \\
\hline & $\mathrm{n}^{0}$ & $\%$ & $\mathrm{n}^{0}$ & $\%$ & & & \\
\hline$<50$ & 111 & 70,7 & 16 & 14,6 & - & 1,00 & - \\
\hline$\geq 50-10.000$ & 38 & 24,2 & 46 & 41,8 & 0,00 & 0,12 & $0,06-0,25$ \\
\hline$>10.000$ & 8 & 5,1 & 48 & 43,6 & 0,00 & 0,02 & $0,01-0,06$ \\
\hline
\end{tabular}

HIV: vírus da imunodeficiência humana, RNA: ácido ribonucléico, P: probabilidade de significância, OR: razão de chances, IC: intervalo de confiança $95 \%$

Queixas otoneurológicas. Dos 162 pacientes tratados com anti-retrovirais, 14 (8,6\%) relataram queixas otoneurológicas e, dos 122 pacientes não tratados, sete $(5,8 \%)$ apresentaram essas queixas $(\mathrm{P}=0,48)$. Os tipos de queixas otoneurológicas estão dispostos na Tabela 2. Perda auditiva com ou sem zumbido representou 78,5\% (11/14) das queixas no tratado e 57,1\% (4/7) no grupo não tratado.

Doenças otoneurológicas. Excluindo-se lesões otológicas prévias à infecção pelo HIV, como perda auditiva induzida por ruído (PAIR) e otite crônica, observaram-se três $(1,8 \%)$ casos de perda auditiva neurossensorial idiopática observadas unicamente no grupo tratado com anti-retrovirais e que surgiram após o início do tratamento (Tabela 3).

Esquemas terapêuticos. Trinta e nove diferentes esquemas terapêuticos foram usados no grupo de 162 pacientes tratados com anti-retrovirais. Os três mais utilizados foram: zidovudinalamivudina-efavirenz $(40,1 \%)$; zidovudina-lamivudina-nevirapina $(7,4 \%)$ e zidovudina-didanosina-nelfinavir $(6,8 \%)$. Os cinco 
Tabela 2 - Distribuição comparativa de 14 pacientes infectados pelo HIV tratados com anti-retroviral e 7 não tratados quanto à queixa otoneurológica. Belo Horizonte, Centro de Treinamento e Referência em Doenças Infecciosas e Parasitárias Orestes Diniz, 2005-2006.

\begin{tabular}{|c|c|c|c|c|c|}
\hline \multirow{4}{*}{ Queixa otoneurológica } & \multicolumn{4}{|c|}{ Pacientes infectados pelo HIV } & \multirow{4}{*}{$\mathrm{P}$} \\
\hline & \multirow{2}{*}{\multicolumn{2}{|c|}{$\begin{array}{c}\text { tratados com } \\
\text { anti-retrovirais } \\
\left(\mathrm{n}^{0}=162\right)\end{array}$}} & \multirow{2}{*}{\multicolumn{2}{|c|}{$\begin{array}{c}\text { não tratados } \\
\text { com anti-retrovirais } \\
\left(\mathrm{n}^{\mathrm{o}}=122\right)\end{array}$}} & \\
\hline & & & & & \\
\hline & $\mathrm{n}^{0}$ & $\%$ & $\mathrm{n}^{0}$ & $\%$ & \\
\hline Perda auditiva e zumbido & 7 & 4,3 & 1 & 0,8 & 0,07 \\
\hline Perda auditiva & 4 & 2,5 & 3 & 2,5 & 0,63 \\
\hline Zumbido & 1 & 0,6 & 3 & 2,5 & 0,22 \\
\hline Tontura & 2 & 1,2 & 0 & 0,0 & 0,31 \\
\hline Total & 14 & 8,6 & 7 & 5,8 & 0,48 \\
\hline
\end{tabular}

HIV: vírus da imunodeficiência humana, P: probabilidade de significância.

Tabela 3 - Distribuição comparativa dos 11 pacientes infectados pelo HIV tratados com anti-retroviral e 4 não tratados em relação ao diagnóstico otológico. Belo Horizonte, Centro de Treinamento e Referência em Doenças Infecciosas e Parasitárias Orestes Diniz, 2005-2006.

\begin{tabular}{|c|c|c|c|c|c|}
\hline \multirow{3}{*}{ Diagnóstico otológico } & \multicolumn{4}{|c|}{ Pacientes infectados pelo HIV } & \multirow{3}{*}{$\mathrm{P}$} \\
\hline & \multicolumn{2}{|c|}{$\begin{array}{c}\text { tratados com } \\
\text { anti-retrovirais } \\
\left(\mathrm{n}^{0}=162\right)\end{array}$} & \multicolumn{2}{|c|}{$\begin{array}{c}\text { não tratados } \\
\text { com anti-retrovirais } \\
\left(n^{\circ}=122\right)\end{array}$} & \\
\hline & $\mathrm{n}^{0}$ & $\%$ & $\mathrm{n}^{0}$ & $\%$ & \\
\hline PAIR & 3 & 1,8 & 2 & 1,6 & $\overline{0,62}$ \\
\hline Otosclerose & 2 & 1,3 & 0 & 0,0 & 0,32 \\
\hline PANS idiopática & 3 & 1,8 & 0 & 0,0 & 0,18 \\
\hline Otite crônica & 1 & 0,6 & 0 & 0,0 & 0,56 \\
\hline Total & 9 & 5,5 & 2 & 1,6 & - \\
\hline
\end{tabular}

HIV: vírus da imunodeficiência humana, P: probabilidade de significância, PAIR: perda auditiva induzida por ruído, PANS: perda auditiva neurossensorial.

esquemas que se associaram aos sintomas otoneurológicos foram: didanosina-lamivudina-lopinavir/r; zidovudina-lamivudinaefavirenz; zidovudina-lamivudina-nevirapina; estavudinalamivudina-lopinavir/r; zidovudina-didanosina-nelfinavir.

Sete drogas compuseram os quatro esquemas associados com perda auditiva neurossensorial encontrados neste estudo, sendo essa correlação mostrada na Tabela 4.

Em relação a outros efeitos colaterais otoneurológicos, dois $(14,2 \%)$ pacientes relataram cabeça oca em uso de efavirenz.

Tabela 4 - Correlação estratificada para cada anti-retroviral em relação à perda auditiva neurossensorial. Belo Horizonte, Centro de Treinamento e Referência em Doenças Infecciosas e Parasitárias Orestes Diniz, 2005-2006.

\begin{tabular}{|c|c|c|c|c|c|}
\hline \multirow[t]{2}{*}{ Anti-retrovirais } & \multicolumn{2}{|c|}{ Usuários } & \multirow{2}{*}{$\begin{array}{l}\text { Perda auditiva } \\
\text { neurossensorial }\end{array}$} & \multirow[b]{2}{*}{$\mathrm{P}$} & \multirow[b]{2}{*}{ OR (IC) } \\
\hline & $\mathrm{n}^{0}$ & $\%$ & & & \\
\hline Lamivudina & 142 & 87,6 & 4 & 0,58 & - \\
\hline Zidovudina & 126 & 77,7 & 2 & 0,21 & $0,27(0,03-2,85)$ \\
\hline Efavirenz & 77 & 47,5 & 1 & 0,34 & $0,36(0,01-4,00)$ \\
\hline Didanosina & 27 & 16,6 & 1 & 0,52 & $1,69(-)$ \\
\hline Lopinavir/r & 24 & 14,8 & 2 & 0,10 & $6,18(0,58-65,85)$ \\
\hline Nelfinavir & 18 & 11,1 & 1 & 0,37 & $2,76(-)$ \\
\hline Estavudina & 16 & 9,8 & 1 & 0,34 & $3,18(-)$ \\
\hline
\end{tabular}

P: probabilidade de significância, OR: razão de chances, IC: intervalo de confiança 95\%.

\section{DISCUSSÃO}

Amostra populacional. Comparando-se o tamanho desta amostra $(\mathrm{N}=284)$ com outros estudos em que há sugestão de ototoxicidade dos anti-retrovirais, observou-se que a casuística do presente estudo foi expressiva. Pesquisas descritivas utilizaram amostragem que variou de 30 a 99 pacientes $^{417}$.

Características epidemiológicas. Os grupos foram semelhantes em relação ao sexo, com predomínio do masculino em ambos os grupos, o que reproduz o padrão epidemiológico da população infectada pelo HIV ${ }^{1924}$. A maior idade do grupo tratado pode ser justificada pelo provável maior tempo de infecção. A diferença encontrada entre os grupos em relação ao tempo de confirmação sorológica corrobora essa hipótese $(\mathrm{P}=0,00)$. A evolução natural da doença também justificaria a diferença ${ }^{1626}$.

Contagem de linfócitos CD4+ e carga viral. Os grupos foram semelhantes em relação à contagem de $\mathrm{CD} 4+(\mathrm{P}=0,60)$. Quando se analisou a carga viral, observou-se que esta foi menor que 10.000 cópias/ml para a maioria dos pacientes em uso de anti-retroviral, quando comparado ao grupo que não estava em uso desse tipo de medicação (Tabela 1). Por outro lado, esses pacientes poderiam estar sob a ação ototóxica das drogas antiretrovirais.

Manifestações otoneurológicas. Para o grupo que não estava sendo tratado, esse raciocínio faz crer que a causa das queixas e doenças otoneurológicas presentes no grupo não tratado estariam associados à ação do vírus, uma vez que esses pacientes estavam com carga viral mais elevada. Para controlar esse possível fator de confusão das manifestações otoneurológicas poderem ser causadas tanto pela ação do vírus quanto pelo tratamento anti-retroviral, a situação ideal seria um grupo de pacientes infectados pelo HIV não tratados e com carga viral abaixo de 50 cópias/ml. Contudo, esse perfil imunológico não é observado de forma freqüente nesses pacientes, o que dificulta um tamanho de amostra suficiente para a comparação.

Em relação à frequiência de queixas otoneurológicas, quatro estudos publicados apresentam frequiência que variou de $26 \%$ a $55 \%$ em pacientes tratados com anti-retrovirais. No presente estudo, não se observou diferença estatisticamente significativa entre as queixas otoneurológicas no grupo tratado $(8,6 \%)$ em relação ao grupo não tratado $(5,8 \%)^{45717}$. Possivelmente, essa diferença de prevalências deveu-se às diferentes formas de seleção dos pacientes e tamanhos das amostras dos estudos revisados. Há, por outro lado, publicações que observaram resultados semelhantes aos encontrados no presente estudo ${ }^{1420}$.

Autores avaliaram os diagnósticos otoneurológicos em estudo descritivo de 106 pacientes brasileiros infectados pelo HIV e revelaram prevalência de 6,6\% de alterações otológicas, não especificando sobre tratamento ${ }^{20}$. Se considerarmos, no presente estudo, as doenças otológicas observadas (Tabela 3) e excluindo-se os exames normais, encontraremos prevalência de $6,8 \%$ de alterações otológicas no grupo tratado com terapia anti-retroviral altamente ativa e 3,2\% no grupo não tratado ou de $10 \%$ para toda a amostra. 
Aqueixa de tontura não parece ter sido um problema relacionado ao uso de drogas anti-retrovirais. A prevalência encontrada foi de $1,2 \%$, concordando com estudos publicados que avaliaram tontura na mesma população aqui estudada ${ }^{14}$. Mesmo com efavirenz, droga que tem sido associada com tontura, principalmente matinal, esse sintoma não foi expressivo ${ }^{18} 28$. Possibilidades para justificar esse achado são: tontura ocorrer somente no início do tratamento; pacientes que suspenderam a medicação por intolerância não fariam parte da amostra avaliada. Quando se considera que Efavirenz estava no esquema utilizado por $40,1 \%$ dos pacientes em uso regular de medicação, pode-se concluir que os efeitos de tontura ocorrem apenas no início do tratamento e esta parece ser uma droga bem tolerada no longo prazo.

Em relação ao diagnóstico otoneurológico, as perdas auditivas dos pacientes não tratados com anti-retrovirais foram desencadeadas por fatores comuns aos quais a população em geral está exposta, como rolha de cerume e ruído ocupacional ${ }^{315}$. Já no grupo tratado com terapia anti-retroviral (Tabela 3), observou-se ocorrência de três perdas auditivas não relacionadas à rolha de cerume ou exposição ao ruído ocupacional e dois casos de otosclerose. Como as perdas auditivas sugiram após o início da terapia anti-retroviral, é possível ter havido influência do uso dos anti-retrovirais no surgimento e/ou desencadeamento da perda auditiva ${ }^{8}{ }^{13}$ 27. Contudo, não se verificou associação estatística dos anti-retrovirais com perda auditiva (Tabela 4). No caso do otosclerose, observada unicamente no grupo tratado, o tamanho amostral talvez possa ter interferido no resultado encontrado, de modo que com maior número de pacientes a correlação com anti-retrovirais talvez pudesse ter sido demonstrada. Mais estudos seriam necessários para esclarecer essa questão.

Esquemas terapêuticos. Conjecturas podem ser feitas em relação a real causa das manifestações audiológicas observadas no grupo tratado com anti-retrovirais: maior tempo de infecção pelo HIV; uso de outras drogas ototóxicas ${ }^{22}$; ; ou terapia antiretroviral. A replicação viral estava controlada, visto que a maioria dos pacientes tratados tinha carga viral menor que 50 cópias por ml de plasma sangüíneo (Tabela 1). 0 uso de drogas ototóxicas não faria parte de possíveis fatores associados, visto que todos os pacientes negaram usar tais medicamentos.

Em conclusão, manifestações audiológicas foram mais freqüentes do que as vestibulares nos pacientes tratados com antiretrovirais. Correlação significativa entre ototoxidade e terapia anti-retroviral não foi confirmada no presente estudo.

\section{REFERÊNCIAS}

1. Calabresi P, Chabner BA. Quimioterapia das doenças neoplásicas. In: Gilman AG (ed) As bases farmacológicas da terapêutica. 9a edição. McGraw-Hill Interamericana, Rio de Janeiro, p. 903-949, 1997.

2. Campanini A, Marani M, Mastroianni A, Cancellieri C, Vicini C. Human immunodeficiency vírus infection: personal experience in changes in head and neck manifestations due to recent antiretroviral therapies. Acta Otorhinolaryngologica Italica 25: 30-35, 2005.

3. Campos CAH, Costa HOO. Tratado de Otorrinolaringologia. Volume 2, Editora Roca, São Paulo, 2002.

4. Castro MN, Bango YM, De Ureta TP, Garcia-Lomas VM, Lopes GF. Hearing loss and human immunodeficiency virus infection. Study of 30 patients. Revista Clínica Española 200: 271-274, 2000.
5. Ceccarelli JC, Maia RA, Floriano SL, Lemos M, Bonaldi LV. Avaliação otoneurológica em pacientes HIV positivos. Revista Brasileira de Otorrinolaringologia 63: 312316, 1997.

6. Chambers HF, Sande M. Fármacos antimicrobianos - Os aminoglicosídeos. In: Gilman AG (ed) As bases farmacológicas da terapêutica. 9a edição. McGrawHill Interamericana, Rio de Janeiro, p. 812-825, 1997.

7. Chandrasekhar SS, Connelly PE, Brahmbhatt SS, Shah CS, Kloser PC, Baredes S. Otologic and audiologic evaluation of human immunodeficiency virus-infect patients. The American Journal of Otology 21: 1-9, 2000.

8. Cheng A, Cunningham LL, Rubel E. Mechanisms of hair cell death and protection. Current Opinion in Otolaryngology \& Head and Neck Surgery 13: 343-348, 2005.

9. Chinnery PF, Elliott C, Green GR, Ress A, Coulthard A, Turnbull DM, Griffiths TD. The spectrum of hearing loss due to mitochondrial DNA defects. Brain: a Journal of Neurology 123: 82-92, 2000.

10. Davis LE, Rarey KE, Mclaren LC. Clinical viral infections and temporal bone histologic studies of patients with AIDS. Otolaryngology-Head and Neck Surgery 113: 695-701, 1995.

11. Fantry LE, Staecker H. Vertigo and Abacavir. Aids Patient Care and Stds 16: 5-7, 2002

12. Grimaldi LM, Luzi L, Martinho GV, Furlan R, NemnI R, Antonelli A, Canal N, Pozza G. Bilateral eighth cranial nerve neuropathy in human immunodeficiency virus infection. Journal of Neurology 240: 363-366, 1993.

13. Lehtonen MS, Moilanen JS, Majamaa K. Increased variation in mtDNA in patients with familiar sensorineural hearing impairment. Human Genetics 113: 220-227, 2003

14. Lignani Júnior L. Avaliação da Aderência aos anti-retrovirais em amostra de pacientes acompanhados no centro de treinamento e referência em Doenças Infecciosas e Parasitárias. Dissertação de mestrado, Universidade Federal de Minas Gerais, Belo Horizonte, BH, 2000.

15. Lopes Filho 0. Deficiência auditiva. In: Ferreira LP (ed) Tratado de Fonoaudiologia. Editora Roca, São Paulo, p. 1-24, 1996.

16. Marques AR, Mansur H. Manifestações Clinicas. In: Focaccia $\mathrm{R}$ (ed) Tratado de Infectologia $2^{a}$ edição, Editora Atheneu, São Paulo, volume 1, p. 123-126, 1998

17. Marra CM, Wechkin HÁ, Longstreth Junior WT, Ress TS, Syapin CL, Gates GA. Hearing loss and antiretroviral therapy in patients infected with HIV-1. Archives of Neurology 54: 407-410, 1997.

18. Ministério da Saúde. Programa de Prevenção a Doenças Sexualmente transmissíveis e AIDS. Recomendações para Terapia Anti-Retroviral em Adultos e Adolescentes Infectados pelo HIV. Disponível em: http://www.aids.gov.br/data/ Pages/LUMIS40967FB4ITEMIDB2E97513C 1694C78954D37DA983FE73EPTBR IE.htm. Acessado em 18/11/2004, Brasília, 2004.

19. Ministério da Saúde. Programa de Prevenção a Doenças Sexualmente transmissíveis e AIDS. Boletim Epidemiológico. Disponível em http//www.aids. gov.br/data/pages/ LUMISD3352823PTBRIE.html> acessado em 28/12/2005, Brasília, 2005.

20. Miziara IV, Valentini JM. Doença de causa otorrinolaringológica em pacientes com AIDS. Jornal Brasileiro de Medicina 76: 24-34, 1999.

21. Nadol Junior JB, Merchant SN. Histopathology and molecular genetics of hearing loss in human. International Journal of Pediatric Otohinolaryngology 61: 1-15, 2001.

22. Palomino J, Pachón P. Aminoglucósidos. Enfermedades Infecciosas y Microbiología Clínica 21: 105-115, 2003.

23. Prasad HKC, Bhojwani KM, Shenoy V, Prasad SC. HIV manifestations in otolaryngology. American Journal of Otolaryngology - Head and Neck Medicine and Surgery 27: 179-185, 2006.

24. Rouquayrol MZ, Façanha MC, Vera FMF. Aspectos epidemiológicos nas doenças transmissíveis. In: Rouquayrol MZA, Filho N (eds) Epidemiologia \& Saúde. Editora Medsi, Rio de Janeiro, p. 213-258, 2003.

25. Rybak LP, Whitworth CA. Ototoxicity: therapeutic opportunities. Drug Discovery Today 10: 1313-1321, 2005.

26. Saag MS. Natural history of HIV-1 disease. In: Broder K (ed) Textbook of AIDS medicine. Willians \& Willians, Baltimore, p. 45-52, 1994. 
27. Seidman MD, Bai U, Khan MJ, Murphy MP, Quirk WS, Castora F, Hinojosa R. Association of mitocondrial DNA deletions and cochlear pathology: a molecular biologic tool. The Laryngoscope 106: 777-783, 1996.

28. Silva P. Drogas antivirais. In: Silva P (ed) Farmacologia. 6a edição. Editora Guanabara Koogan, Rio de Janeiro, p. 1128-1140, 2002.

29. Simdon J, Walters D, Bartlett S, Connick E. Ototoxicity associated with use of nucleoside analog reverse transcriptase inhibitors: a report of
3 possible cases and review of the literature. Clinical Infectious Diseases 32: 1623-1627, 2001.

30. Tseng AL, Dolovich L, Salit IE. Azitromicin-related ototoxicity in patients infected with human immunodeficiency virus. Clinical Infectious Diseases 24: 76-77, 1997.

31. Vogeser M, Colebunders R, Depraetere K, Van Wanzeele P, Van Gehuchten S. Deafness caused by didanosina. European Journal of Clinical Microbiology \& Infectious Diseases 17: 214-215, 1998. 